\title{
An Innovative User Interface Concept for Large Hierarchical Data Spaces by Example of the EPDM Domain
}

\author{
Fredrik Gundelsweiler, Robert Konstanzer, Harald Reiterer \\ Human-Computer Interaction Lab, University of Konstanz \\ Universitätsstrasse 10, Box D73 \\ 78457 Konstanz, Germany \\ \{Fredrik.Gundelsweiler, Robert.Konstanzer, Harald.Reiterer\}@uni-konstanz.de \\ +497531883547
}

\begin{abstract}
In this paper we will describe a new approach to navigation and interaction with the visualization of a data space of an electronic product data management (EPDM) system. The main problems are the amount of data and therefore the location of an item of interest, show specific relations and narrow or expand the information space. We combine a scalable visualization of the hierarchical data space with search and filter techniques like dynamic queries and direct manipulation to solve the stated problems. We assume that the proposed concept for EPDM can be adapted to data spaces like social networks or company structures.
\end{abstract}

Author Keywords

Scalable visualization, hierarchies, semantic pixel display.

ACM Classification Keywords

H5.m. Information interfaces (e.g., HCI): Miscellaneous.

\section{INTRODUCTION}

Many data structures in the real world can be visualized by hierarchies or networks. Examples for this are the social relations between users of an online community, the hierarchical structures in companies or the "is part of" relations of a product and its sub components. There are many approaches to the visualization of hierarchies and networks in the research community. Our approach explicitly deals with the visualization of EPDM data spaces and the tasks of their users. Nevertheless, we assume that our concept can be assigned to other domains.

Today companies are trying hard to reduce the time to market of a product, reduce documentation and change management effort, decrease repair effort, and reduce the risk with regards to international product liability. The vision is that with the introduction of an EPDM system throughout the whole product lifecycle, companies can draw nearer to these goals. We focus on the product maintenance phase where employees have to maintain the product's data.

The analysis of conventional EPDM systems ${ }^{1}$ reveals essential weaknesses. Often there is no overview and no adequate narrowing of the information space. Other poor solutions are static result displays, bad filter options and pop up windows. Mostly navigation is implemented in the same way as in Microsoft Windows Explorer. We therefore break up with conservative UIs and demonstrate the value of advanced UIs that use the screen space optimally and can be adapted to the user's needs. This is a difficult task because the amount of data can exceed several hundred thousand items, each item consisting of its own attributes and relations.

\section{DATA SPACE}

The structure of our exemplary data space is the one of a computer manufacturing company. The data space consists of the components product, part, variant, basis, network, hardware, software, cpu, memory, connector, software module and pin. These components all have different variants which are interconnected and thus grouped.

In reality the amount of data varies depending on the domain. A company producing a simple product like a shovel would have a less complex data space structure, but companies producing cars or even airplanes would have a much more complex data space. Here we use a recursive data generation program to create a random data space with the amount of 7.500 up to 100.000 data items.

\section{USER TASKS}

In the requirements analysis we found out that the tasks of EPDM system users vary widely, therefore we focus on their main tasks: create a new object, edit an object, and

\footnotetext{
${ }^{1}$ Common EPDM systems are IBM Enovia; $3 D S$ Enovia

MatrixOne; mySAP PLM, and UGS TeamCenter.
} 
find specific objects. There are some other tasks like the comparison of objects and their relations, and the check and adaptation of relations, which we will take into account in an enhanced version of our prototype.

\section{VISUALIZATION AND NAVIGATION}

Figure 1 shows the user interface of our interactive prototype, which we realized with java and the prefuse framework $^{2}$. It consists of an object filter and semantic visualization area for each object category.

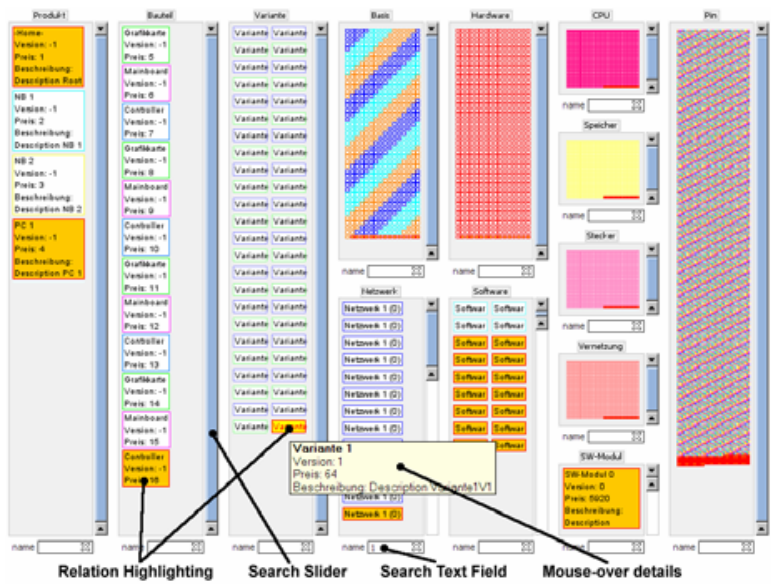

Figure 1: Prototype application with about 15.000 items.

The user interface uses a semantic zoom rendering to adapt the amount of visible information to the screen size of an object. This makes it possible to show only a few objects or even several thousands of objects in a pixel display [2] in each area. The more screen space available for one object, the more information of the object can be shown to the user (compare e.g. the product area on the left and pin area on the right in Figure 1). In contrast to most graph visualizations, the complexity of our user interface is independent of the amount of objects to be displayed. With a larger data amount the displayed objects in the areas are drawn with a higher resolution, but the complexity of the user interface is the same. The border colour of objects is mapped to its name attribute to let the users locate the different name areas at a glance.

\section{FILTER, SEARCH AND NAVIGATION}

Further problems of EPDM systems are to find a specific object, to see its overall dependencies, and to navigate through the information space. Therefore our concept follows the mantra of information seeking of "overview first, zoom and filter, then details on demand." [3]. We propose four techniques to improve access to the data space and solve the described problems. The users can set a focus on interesting object areas to get a first overview over the amount of data. Per navigation and filter, users can narrow down or expand the data space and thus are able to get more

\footnotetext{
${ }^{2}$ Prefuse (URL: http://prefuse.org, last visited 03.12.2007).
}

information about relevant objects and remove irrelevant ones from the visualization. Users always stay in context, but are able to view dependencies by highlighting and some details by mouse over. They can work with the objects by opening a context menu for each object with a right click. The interactive filter and visualization areas are integrated into the filter process and in turn the presentation of search results is integrated into the graph visualization. The users can directly query a search by editing a text field or start a filter action by using the dynamic query [1] slider. The result is shown in the same screen area with immediate feedback. For the moment the slider is mapped onto the object attribute price. In the future we will provide a combo box for the attribute selection. This simple but powerful filter concept supports straight forward queries for each object area. The users can analyze the interdependencies between objects by highlighting and thus detecting the impact of both a filter action and/or a navigation step on all object areas. This allows complex queries on the data space without an overloaded graphical user interface and complex query languages. A left click on an object narrows down the information space to all related objects. This navigation concept is integrated into the filter concept to enable the users to narrow the results by selecting specific objects. We produced a demo video which is available on our homepage $^{3}$ to facilitate the understanding of our concepts.

\section{OUTLOOK AND CONCLUSION}

We developed a visualization that uses the screen space in an efficient way. The overview over the whole data space supports a direct access to sub information areas as well as specific objects and their relations. Navigation, search and filter techniques help users to narrow down the information space to the relevant data set. These techniques enable users to adjust the user interface to match the current task and are not as laborious as in conventional EPDM systems. We assume that our concept solves important navigation, visualization and interaction problems of EPDM systems.

\section{REFERENCES}

1. Ahlberg, C.; Williamson, C. \& Shneiderman, B.: Dynamic queries for information exploration: an implementation and evaluation, in 'CHI '92: Proceedings of the SIGCHI conference on Human factors in computing systems', ACM Press, New York, NY, USA, pp. 619 626, 1992.

2. Keim, D. A.: Pixel oriented Database Visualizations, in 'SIGMODRECORD special issue on 'Information Visualization', 1996.

3. Shneiderman, Ben: The Eyes Have It: A Task by Data Type Taxonomy for Information Visualizations. In Proceedings of the IEEE Symposium on Visual Languages, pages 336 343, Washington. IEEE Computer Society Press, 1996.

\footnotetext{
${ }^{3}$ HCI Konstanz (URL: http://hci.uni konstanz.de/epdm)
} 